\title{
SINGLE-MOLECULE PROTEIN CONFORMATIONAL DYNAMICS UNDER ENZYMATIC REACTIONS AND CELL SIGNALING
}

Yufan He, Saptarshi Mukherjee, H. Peter Lu*

Bowling Green State University, Department of Chemistry, Center for Photochemical Sciences, Bowling Green, $\mathrm{OH} 43403$; *hplu@bgsu.edu

Enzymatic reactions are traditionally studied at the ensemble level, despite significant static and dynamic inhomogeneities. Subtle conformational changes play a crucial role in protein functions, and these protein conformations are highly dynamic rather than being static. We applied AFM-enhanced single-molecule spectroscopy to study the mechanisms and dynamics of enzymatic reactions involved with kinase and lysozyme proteins. Enzymatic reaction turnovers and the associated structure changes of individual protein molecules were observed simultaneously in real-time by single-molecule FRET detections. We obtained the rates for single-molecule conformational active-site open-close fluctuation and correlated enzymatic reactions. We have demonstrated a specific statistical analysis to reveal single-molecule FRET anti-correlated fluctuations from a high background of fluorescence correlated thermal fluctuations. Our new approach is applicable to a wide range of single-molecule FRET measurements for protein conformational changes under enzymatic reactions. 